\title{
Efectos de la Aspirina en Fetos de Rata con Reducción de la Presión de la Perfusión Uterina
}

\author{
Effects of Aspirin on Rat Fetuses with Reduction of Uterine Perfusion Pressure
}

\author{
Daniel Conei ${ }^{1,2,3}$; Ditsa Cáceres Gutiérrez ${ }^{1}$; Michael Haverbeck³; Roberto Fierro; \\ Carlos Veuthey ${ }^{4}$; Mariano del Sol${ }^{2} \&$ Mariana Rojas Rauco ${ }^{1}$
}

\begin{abstract}
CONEI, D.; CÁCERES, G. D.; HAVERBECK, M.; FIERRO, R.; VEUTHEY, C.; DEL SOL, M. \& ROJAS, R. M. Efectos de la aspirina en fetos de rata con reducción de la presión de la perfusión uterina. Int. J. Morphol., 37(2):739-743, 2019.

RESUMEN: La preeclampsia (PE) es un trastorno hipertensivo inducido por el embarazo donde se reduce la presión de la perfusión uterina. Investigaciones avalan el uso de dosis baja de aspirina (DBAAS) y su utilidad en la prevención de PE en gestantes con factores de riesgo. Sus beneficios en modelos animales sometidos a esta reduccción no están determinados. El objetivo de la investigación fue analizar la presión arterial sistémica y los hallazgos morfológicos a nivel renal en fetos de ratas con reducción de la presión de perfusión uterina (RPPU) expuestas a DBAAS en comparación a las no expuestas. Se conformaron cuatro grupos de ratas hembras preñadas Sprague Dawley (n=5). A los 14,5 días post-concepción (dpc), vía quirúrgica se indujo RPPU, ligando arterias uterinas, conformándose el grupo RPPU y el grupo RPPU+DBAAS al que se le administró $5 \mathrm{mg} / \mathrm{kg} / \mathrm{día}$ de aspirina vía oral. El grupo control lo conformaron las no operadas y el grupo DBAAS se le administró aspirina en igual dosis desde el 14,5 dpc. A los 18,5 dpc, previo a la eutansia se midió la presión arterial sistémica con pletismógrafo caudal Insight v2.11 y se extrajeron los fetos. Se midió la longitud céfalo-caudal (LCC), se procesaron y tiñeron con hematoxilina-eosina, describiéndose cortes histológicos transversales a nivel renal. Se determinó que en la presión arterial media, hubo diferencias significativas entre el grupo RPPU y RPPU+DBAAS (p<0,05). El tamaño de los fetos fue menor en el grupo RPPU ( $<<0,0001)$, donde 1 feto presentó hernia umbilical congénita. La cuantificación de vesículas renales también fue menor ( $\mathrm{p}<0,005)$. En conclusión, la administración de DBAAS disminuye los efectos inducidos por la RPPU en cuanto al tamaño fetal, morfología renal y malformaciones congénitas como hernia umbilical. En cuanto a la presión arterial sistémica, tendría efectos sólo en presión arterial media.
\end{abstract}

PALABRAS CLAVE: Preeclampsia; Hipertensión inducida por el embarazo; Presión arterial; Aspirina.

\section{INTRODUCCIÓN}

La preeclampsia (PE) y los trastornos hipertensivos relacionados a la gestación poseen tasas de incidencia en los Estados Unidos, Canadá y la Europa Occidental, que van desde el 2 al $5 \%$ (Bokslag et al., 2016). En América Latina, del total de muertes maternas entre 2003 y 2009, el $22,1 \%$ de ellas correspondió a trastornos hipertensivos, siendo superado solo por las hemorragias (Say et al., 2014).

Dentro de los síndromes hipertensivos, la PE es la más estudiada, por ser una de las condiciones que presenta mayor riesgo de morbimortalidad para la madre y el feto. Esta se determina por el aumento de la presión arterial en valores sobre $140 \mathrm{mmHg}$ de presión sistólica y sobre 90 $\mathrm{mmHg}$ de presión diastólica, asociada a proteinuria que se desarrolla generalmente desde las 20 semanas de gestación (Ministerio de Salud, 2015). Sin embargo, también se puede asociar a otra sintomatología como insuficiencia renal o daño hepático (Fushima et al., 2016). Su patogenia está asociada a alteraciones en la placentación, con un remodelamiento vascular incompleto a nivel de las arterias espiraladas, que produce una disminución del flujo úteroplacentario, generando un estrés oxidativo que favorece la liberación de citoquinas proinflamatorias y la activación del endotelio donde disminuye la producción de prostaglandinas y óxido nítrico, y aumenta la producción de endotelina, dando como resultado una reducción del flujo placentario y un ambiente hipóxico patológico para el feto (Henderson et al., 2017).

\footnotetext{
${ }^{1}$ Laboratorio de Embriología Comparada, Programa de Anatomía y Biología del Desarrollo, Instituto de Ciencias Biomédicas, Facultad de Medicina, Universidad de Chile, Santiago, Chile.

${ }^{2}$ Programa de Doctorado en Ciencias Morfológicas, Universidad de La Frontera, Temuco, Chile.

${ }^{3}$ Departamento de Ciencias Morfológicas, Facultad de Medicina y Ciencia, Universidad San Sebastián, Puerto Montt, Chile.

${ }^{4}$ Centro de Investigación en Ciencias Odontológicas, Facultad de Odontología, Universidad de La Frontera, Temuco, Chile.
} 
En la embriogénesis, existe un estado hipóxico fisiológico que genera la activación de genes que inducen la formación del sistema circulatorio fetal. Si es un estado crónico, se transformará en una hipoxia patológica, y si es tolerado por el feto, se generará una redistribución del flujo sanguíneo, manteniendo el aporte de oxígeno al sistema nervioso central y corazón, en desmedro de una disminución del flujo sanguíneo hacia riñones, hígado, músculo esquelético y otros tejidos, provocando un retardo en el crecimiento y alteraciones en la función orgánica (Steegers et al., 2010). Se ha observado que órganos como el riñón presentan un menor peso y una disminución en el número de glomérulos, asociado a una hipertrofia de estos (Baserga et al., 2009).

Por años, diversos estudios han intentado probar tratamientos que contrarresten los efectos o eviten la aparición de PE. Dentro de los agentes estudiados, se encuentran el uso de calcio suplementario y aceite de pescado, pero no han tenido los beneficios esperados. Por otro lado, como ya se han probado los beneficios del uso de dosis bajas de aspirina (entre 60 y 150 mg/día) en la hipertensión arterial crónica (Divisón et al., 2012; Roberge et al., 2017; Atallah et al., 2017), cabe preguntarse lo que ocurre con su uso en la prevención de la PE. En el año 2014, la United States Preventive Service Task Force (USPSTF) recomendó el uso de dosis bajas de aspirina antes de las 12 semanas de gestación en mujeres que poseen un alto riesgo de tener PE, reconociendo como estadio de alto riesgo tener historia de $\mathrm{PE}$, restricción del crecimiento intrauterino, parto de pretérmino, desprendimiento de placenta, muerte fetal, diabetes mellitus, hipertensión arterial, doppler de arteria uterina alterado, entre otros (LeFevre \& U.S. Preventive Services Task Force, 2014).

La aspirina o ácido acetilsalicílico pertenece a la familia de los salicilatos y se caracteriza por tener un anillo bencénico unido a un grupo acetilo y un grupo carboxilo, teniendo diversos efectos dependiendo de la dosis. Una dosis baja de ácido acetilsalicílico (DBAAS) por día, genera un efecto antiplaquetario a través de la acetilación de la enzima ciclooxigenasa plaquetaria (COX-1) y la inhibición de la producción de tromboxano A2, que además de ser un potente agregante plaquetario produce vasoconstricción (Sáez \& Carvajal, 2012; Cadavid, 2017). También posee acciones antiinflamatorias cuyo mecanismo involucra la síntesis de lipoxinas o ATL (Aspirin-Triggered Lipoxin). Las lipoxinas promueven la acción antiinflamatoria inhibiendo la interacción entre el endotelio y los leucocitos, como también poseen poder antioxidante, bloqueando la fabricación de especies reactivas de oxígeno (ROS) en células endoteliales y poseen acciones inmunomoduladoras (Nascimento-Silva et al., 2007). Si bien los estudios son controversiales, tanto la acción antiplaquetaria como la antiinflamatoria de la aspirina serían importantes en la patogenia de la PE, ya que ésta actuaría a nivel del endotelio favoreciendo tanto la disminución de la resistencia de los vasos uteroplacentarios, como de los radicales libres, y como consecuencia, del ambiente hipóxico intrauterino patológico (Sáez \& Carvajal).

Sumando estos factores, nuestro objetivo de estudio fue analizar la presión arterial sistémica y los hallazgos morfológicos en fetos de ratas con reducción de la presión de perfusión uterina (RPPU) vía quirírugica expuestas a DBAAS en comparación a las no expuestas, con el fin de probar la hipótesis de que los fetos sometidos a un ambiente hipóxico y que no fueron expuestos a DBAAS, presentan mayor magnitud de eventos asociados a la hipoxia que aquellos que sí fueron expuestos.

\section{MATERIAL Y MÉTODO}

Se realizó un estudio de tipo experimental aleatorizado, longitudinal y prospectivo el cual fue llevado a cabo en el Centro de Excelencia en Estudios Morfológicos y Quirúrgicos de la Universidad de La Frontera. Se utilizaron 20 ratas de la especie Sprague Dawley, las cuales fueron colocadas junto a un macho distribuidas en jaulas donde había dos hembras por cada macho. Se consideró la presencia de tapón mucoso a nivel vaginal como el instante 0,5 de la gestación.

Luego de esto, se conformaron 4 grupos de manera aleatorizada: grupo control, grupo RPPU, grupo RPPU+DBAAS y grupo DBAAS. Todos los grupos fueron alojados en una jaula individual a $23^{\circ} \mathrm{C}$ en un ciclo de luz oscuridad de $12 \mathrm{~h}$ respectivamente, con agua y alimento adlibitum.

En el día 14,5 post-coital (dpc), las hembras de los grupos RPPU y RPPU+DBAAS fueron sometidas al procedimiento quirúrgico de RPPU, en el cual se induce un estado preeclámptico y un sitio de alta resistencia a través de la ligadura de las arterias uterinas y ováricas (Fushima et al.). Posteriormente, el grupo RPPU+DBASS y grupo DBAAS recibieron $5 \mathrm{mg} / \mathrm{kg} /$ día de aspirina disuelta en agua destilada vía oral, catalogado como dosis mínima en estudios previos (Masuda et al., 2018), desde las $6 \mathrm{~h}$ post-operatorias hasta su eutanasia.

En el 18,5 dpc se midió la presión arterial sistólica (PAS), media (PAM) y diastólica (PAD) de forma no invasiva a las ratas de todos los grupos, con el pletismógrafo de cauda Insight v2.11. Luego se practicó la eutanasia de las ratas con una sobredosis de $180 \mathrm{mg} / \mathrm{kg}$ de tiopental sódico inyectable, intraperitoneal y se extrajeron los fetos. Posterior a su muerte, en una etapa inicial a todos los fetos de cada rata se les midió su longitud céfalo-caudal (LCC), usan- 
do papel milimetrado, bajo una estereolupa (LEICA S6D) con luz LED (LEICA KL300 LED) y se les observaron sus características anatómicas.

Para la observación y descripción de riñones, se seleccionó un feto de cada grupo y fueron fijados en formalina tamponada en PBS al $10 \%$, deshidratados en una batería de orden creciente de alcoholes e incluidos en Paraplast X-TRA ${ }^{\circledR}$. Se obtuvieron cortes transversales seriados a nivel renal por cada feto con microtomo Leica (RM2255) de $5 \mu \mathrm{m}$ de espesor, los cuales fueron adheridos a un portaobjeto y teñidos con hematoxilina-eosina y azul de Alcián. Se observaron los cortes con un microscopio óptico Leica (DM750) con cámara digital Leica (ICC50 HD). Cada 5 muestras histológicas, se localizó la misma zona histológica a nivel renal para cada grupo con el fin de hacer la descripción morfológica y cuantificación de las vesículas renales presentes con el software STEPanizer ${ }^{\circledR}$ Stereological tool, version 1.0.

Se analizaron los resultados a través del programa estadístico GraphPad Prism (GraphPad Software, San Diego, CA, E.E.U.U.), en los cuales se estimó la media de las mediciones antropométricas obtenidas y de fetos abortados en cada grupo de ratas. Para la comparación entre los grupos se utilizó el test de ANOVA y el test de comparación de Tukey o el test de Kruskal Wallis con distribución normal y distribución no normal, respectivamente. Todos los datos fueron expresados como promedio \pm desviación estándar, considerando una diferencia significativa con valor de $\mathrm{p}<0,05$.

\section{RESULTADOS}

En la medición de la presión arterial sistémica, en la presión sistólica (PAS), media (PAM) y diastólica (PAD) hubo diferencias significativas entre los grupos control y RPPU. En la PAS, además hubo diferencias entre el grupo RPPU y DBAAS; y en la PAM entre los grupos RPPU+DBAAS en comparación al grupo RPPU. En relación al tamaño de las ca- madas, el grupo control presentó 60 crías, grupo RPPU 44 crías/ 20 abortos, grupo RPPU+DBAAS 40 crías/13 abortos y grupo DBAAS 55 crías. Un feto del grupo RPPU presentó una hernia umbilical congénita (Fig. 1). En la LCC, el grupo sometido a la intervención quirúrgica presentó un menor tamaño de las crías, arrojando diferencias significativas en comparación al resto de los grupos, situación similar a la presentada en la cantidad de vesículas renales, siendo menor en el grupo RPPU (Tabla I, Fig. 1).

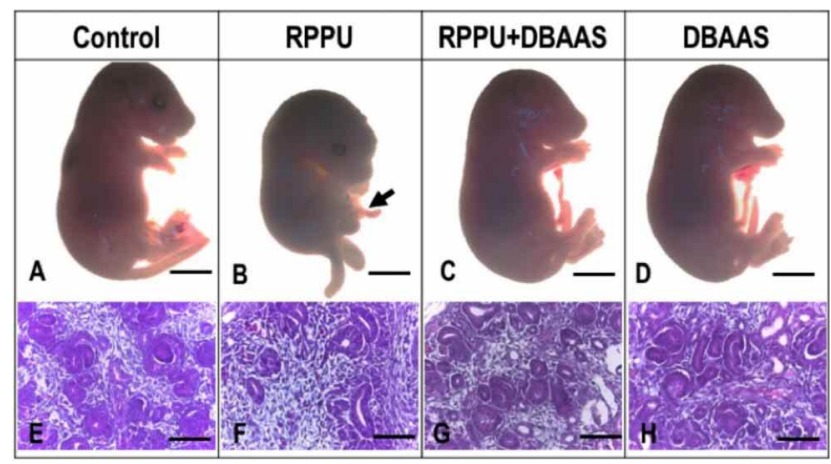

Fig. 1. En A, B, C y D fetos de los respectivos grupos control, RPPU, RPPU+DBAAS y DBAAS. En B, flecha indica hernia umbilical congénita. Barra $5 \mathrm{~mm}$. En E, F, G y H riñones fetales de los respectivos grupos de estudio. Barra $200 \mu \mathrm{m}$.

\section{DISCUSIÓN}

Este estudio fue realizado con el fin de analizar los efectos de la aspirina en fetos de ratas con RUPP a través de la evaluación de diversos eventos asociados a la hipoxia. La muerte fetal y neonatal es uno de los riesgos asociados a los síndromes hipertensivos del embarazo, razón por la cual diversos tratamientos miden su efectividad frente a esta. De acuerdo con el número de fetos totales y el número de abortos, al comparar los promedios de cada grupo, no se encontraron diferencias significativas, por lo que se puede inferir que la aspirina no influiría en estas pérdidas reproductivas.

Tabla I. Descripción de variables cuantificadas entre los grupos de estudio.

\begin{tabular}{lcccc}
\hline \multicolumn{1}{c}{ Variable } & Grupo Control & Grupo RPPU & Grupo RPPU+DBAAS & Grupo DBAAS \\
\hline PAS (mm de Hg) & $150,2 \pm 13,97$ & $190,6 \pm 6,38^{\mathrm{a}, \mathrm{b}}$ & $170,2 \pm 9,36$ & $152,2 \pm 10,05$ \\
PAM (mm de Hg) & $135 \pm 5,24$ & $154,4 \pm 11,30 \mathrm{c}^{\mathrm{d}}$ & $137 \pm 10,51$ & $130,6 \pm 4,21$ \\
PAD (mm de Hg) & $106,8 \pm 7,12$ & $133,2 \pm 4,86 \mathrm{e}$ & $115,4 \pm 10,73$ & $107,4 \pm 6,91$ \\
Número de fetos (n) & 60 & 44 & 40 & 55 \\
Longitud céfalo-nalga (mm) & $26,9 \pm 1,85$ & $15,4 \pm 3,01 \mathrm{f}$ & $23,8 \pm 3,25$ & $25 \pm 2,88$ \\
Número de abortos (n) & - & 20 & 13 & - \\
Número de corpúsculos néfricos (n) & $12,33 \pm 4,27$ & $6,25 \pm 1,46^{\mathrm{g}}$ & $11,46 \pm 3,95$ & $10,76 \pm 5,02$ \\
\hline
\end{tabular}

RPPU: reducción de la presión de perfusión uterina; DBAAS: baja dosis de aspirina; PAS: presión arterial sistólica; PAM: presión arterial media; PAD: presión arterial diastólica; mmHg: milímetros de mercurio; n: número; mm: milímetros. (a): p $<0,005$ entre grupos control y RPPU; (b): p<0,05 entre grupos RPPU y DBAAS; (c): p<0,05 entre grupos control y RPPU; (d): p<0,05 entre grupos RPPU y RPPU+DBAAS; (e): p<0,05 entre grupos control y RPPU; (f): $p<0,0001$ entre todos los grupos en relación al grupo RPPU; (g): $p<0,005$ entre todos los grupos en relación al grupo RPPU. 
En la literatura no hay cifras descritas al respecto. Sin embargo, Fushima et al. menciona en su estudio que la ligadura de los vasos uterinos causa muertes embrionarias significativas, al igual que Faulkner et al. (2016). Por otro lado, en este estudio también se observó que los abortos presentes en cada grupo se encontraban en zonas próximas a la ligadura de vasos ováricos o vasos uterinos, lo que refuerza la idea de que las muertes pueden deberse a la intervención quirúrgica.

Respecto de las cifras de presión arterial, hubo diferencias significativas entre el grupo control y el grupo RPPU, lo que concuerda con los efectos que busca imitar la reducción de la presión de perfusión uterina, incluyendo la hipertensión, y la restricción del crecimiento fetal (Fushima et al.). Diversos estudios han reportado un aumento significativo de la PAM en el grupo sometido RPPU. Faulkner et al. midieron la PAM de forma invasiva el día 19 de gestación registrando en el grupo control una PAM de 102,2 $\pm 3,2 \mathrm{mmHg}$ y en el grupo de ratas RPPU 122,5 $\pm 2,0 \mathrm{mmHg}$. Fushima et al. midieron la PAM un día después de la cirugía con pletismógrafo, demostrando una elevación significativa en sus modelos de rata RPPU versus el grupo control. Por otra parte, en la PAS hubo diferencias significativas entre los grupos control y RPPU, como también entre este último y el grupo al DBAAS. En la PAD sólo se observaron diferencias entre los grupos RPPU y control. Ushida et al. (2016) reportó en su investigación un aumento de las presiones arteriales sistólica y diastólica en modelos de ratas RPPU las cuales fueron medidas el día 19 postconcepción. Esto resulta interesante, pues en la mayoría de los estudios que utilizan modelos de ratas preeclámpticas sólo se mide la PAM porque revela cómo están siendo perfundidos los órganos corporales, pero un aumento de la PAS y la PAD podrían indicar que hay una disminución de la complacencia de la aorta y un aumento de la resistencia vascular periférica, muy similar a lo que ocurre durante la preeclampsia (Brennan et al., 2014). Se puede inferir por lo tanto que la aspirina podría tener efectos sobre la presión arterial media en estos modelos de ratas al existir una disminución de las presiones arteriales en el grupo RPPU+DBAAS, similares al grupo control.

El promedio de la LCC de los fetos fue menor en el grupo RPPU, lo que sugiere que hubo crecimiento restringido asociado a la disminución de la entrega de oxígeno, como se evidencia en otros estudios en los cuales se produce una reducción en el peso y la talla de los fetos (Anderson et al., 2005; Amaral et al., 2015). Esta variación de promedios entre los grupos y la comparación de ellos fue estadísticamente significativa, lo que se debería a que la aspirina podría tener efectos sobre el crecimiento fetal. En una revisión sistemática realizada por Roberge et al., se determinó que la aspirina administrada en mujeres con factores de riesgo para presentar PE previene su aparición, y disminuye la restricción del crecimiento fetal de forma significativa si esta se administra antes de las 16 semanas y con una dosis de $100 \mathrm{mg}$ /día. La aspirina puede contrarrestar los efectos provocados por RPPU dado que actúa frente a la activación endotelial, inhibiendo la producción de tromboxanos, sin provocar la alteración de la secreción de prostaglandinas, como también reduce los efectos del estrés oxidativo (Atallah et al.). Un estudio reciente que buscaba entender el efecto de la aspirina en la prevención de la PE y su relación con el sFlt1, que es el receptor soluble del VEGF y que al unirse a este inhibe su acción angiogénica, determinó un balance entre las sustancias angiogénicas y antiangiogénicas (Li et al., 2015). De esta forma, este fármaco podría contribuir por medio de distintos mecanismos de acción a disminuir los efectos de adaptación fetal a la hipoxia.

El riñón es uno de los órganos donde disminuye el gasto cardíaco durante un proceso hipóxico, por lo que su desarrollo puede verse alterado. Estudios han descrito que los riñones de fetos de ratas sometidos a hipoxia tienen un tamaño y peso menor (Bueno et al., 2010; Plank et al., 2010). Sumado a esto, se ha descrito que existe una hipertrofia de estos que podría ser compensatoria (Baserga et al.). Se ha estimado además que esto podría aumentar el riesgo de desarrollar hipertensión en la adultez (Gonzalez-Rodriguez et al., 2013). En el caso de este estudio, se observó que el riñón del feto sometido a hipoxia presentó una disminución de vesículas renales respecto del grupo control y el grupo RPPU. Es importante profundizar en este punto, ya que esta alteración podría deberse al efecto que genera un ambiente hipóxico y la consecuente reducción del flujo sanguíneo fetal. No se puede estimar en esta descripción si la aspirina tiene efectos a nivel renal que puedan contrarrestar la disminución del gasto cardiaco del riñón. Sin embargo, se pueden realizar estudios sumando a otras técnicas que permitan determinar las diferencias entre los grupos.

En conclusión, la aspirina tuvo efectos beneficiosos en el tamaño fetal, morfología renal y la PAM en las ratas sometidas a RPPU. Es necesario realizar otras técnicas que permitan reconocer otras modificaciones de forma más específica como la neoformación de vasos sanguíneos, la presencia de marcadores de hipoxia, la disminución del gasto cardiaco a nivel renal, entre otras, con el fin de establecer la correlación entre la angiogénesis y el eventual estado hipóxico que induce este modelo de RPPU.

CONEI, D.; CÁCERES, G. D.; HAVERBECK, M.; FIERRO, R.; VEUTHEY, C.; DEL SOL, M. \& ROJAS, R. M. Effects of aspirin on rat fetuses with reduction of uterine perfusion pressure. Int. J. Morphol., 37(2):739-743, 2019

SUMMARY: Preeclampsia (PE) is a hypertensive disorder induced by pregnancy where there is a reduction in the uterine perfusion pressure. Research supports the use of low dose aspirin (LDAAS) and 
its usefulness in the prevention of PE in pregnant women with risk factors. Their benefits in animal models subject to RUPP are not determined. The objective of the investigation was to analyze the systemic blood pressure and the morphological findings at renal level in fetuses of rats with reduction of uterine perfusion pressure (RUPP) exposed to LDAAS compared to those not exposed. Four groups of pregnant female rats Sprague Dawley $(n=5)$ were formed. At 14.5 days post-conception (dpc), surgical RUPP was induced, ligating uterine arteries, with the RUPP group and RUPP+LDAAS group being given $5 \mathrm{mg} / \mathrm{kg} /$ day of aspirin orally. The control group was made up of those not operated and the LDAAS group was administered aspirin in the same dose from $14.5 \mathrm{dpc}$. A $18.5 \mathrm{dpc}$, prior to euthanasia systemic blood pressure was measured with flow plethysmograph Insight v2.11 and fetuses were extracted. The cephalo-caudal length (CCL) was measured, processed and stained with hematoxylin-eosin, describing transverse histological sections at the kidney level. It was determined that in the mean arterial pressure, there were significant differences between the group RUPP and RUPP+LDAAS ( $\mathrm{p}<0.05)$. The size of the fetuses was lower in the RUPP group ( $p<0.0001$ ), where one fetus presented congenital umbilical hernia. The quantification of renal vesicles was also lower $(\mathrm{p}<0.005)$. In conclusion, the administration of LDAAS decreases the effects induced by RUPP in terms of fetal size, renal morphology and congenital malformations such as umbilical hernia. Regarding the systemic blood pressure, effects would only mean arterial pressure.

KEY WORDS: Preeclampsia; Pregnancy induced hypertension; Blood pressure; Aspirin.

\section{REFERENCIAS BIBLIOGRÁFICAS}

Amaral, L. M.; Cornelius, D. C.; Harmon, A.; Moseley, J.; Martin, J. N. Jr. \& LaMarca, B. 17-hydroxyprogesterone caproate significantly improves clinical characteristics of preeclampsia in the reduced uterine perfusion pressure rat model. Hypertension, 65(1):225-31, 2015.

Anderson, C. M.; Lopez, F.; Zhang, H. Y.; Pavlish, K. \& Benoit, J. N. Reduced uteroplacental perfusion alters uterine arcuate artery function in the pregnant Sprague-Dawley rat. Biol. Reprod., 72(3):762-6, 2005.

Atallah, A.; Lecarpentier, E.; Goffinet, F.; Doret-Dion, M.; Gaucherand, P. \& Tsatsaris, V. Aspirin for prevention of preeclampsia. Drugs, 77(17):181931, 2017.

Baserga, M.; Bares, A. L.; Hale, M. A.; Callaway, C. W.; McKnight, R. A.; Lane, P. H. \& Lane, R. H. Uteroplacental insufficiency affects kidney VEGF expression in a model of IUGR with compensatory glomerular hypertrophy and hypertension. Early Hum. Dev., 85(6):361-7, 2009.

Bokslag, A.; van Weissenbruch, M.; Mol, B. W. \& de Groot, C. J. Preeclampsia; short and long-term consequences for mother and neonate. Early Hum. Dev., 102:47-50, 2016.

Brennan, L. J.; Morton, J. S. \& Davidge, S. T. Vascular dysfunction in preeclampsia. Microcirculation, 21(1):4-14, 2014.

Bueno, M. P.; Barini, R.; Gonçalves, F. L.; Veríssimo, R. P. \& Sbragia, L. Experimental rat model for fetal growth restriction: effects on liver glycogen and intestinal and renal morphometry. Rev. Bras. Ginecol. Obstet., 32(4):163-8, 2010.

Cadavid, A. P. Aspirin: the mechanism of action revisited in the context of pregnancy complications. Front. Immunol., 8:261, 2017.

Divisón, J. A.; Galgo, A.; Polo, J. \& Durá, R. Primary prevention with aspirin. Semergen., 38(6):366-76, 2012.

Faulkner, J. L.; Cornelius, D. C.; Amaral, L. M.; Harmon, A. C.; Cunningham, M. W. Jr.; Darby, M. M.; Ibrahim, T.; Thomas, D. S.; Herse, F.; Wallukat, G.; et al. Vitamin D supplementation improves pathophysiology in a rat model of preeclampsia. Am. J. Physiol. Regul. Integr. Comp. Physiol., 310(4):R346-54, 2016

Fushima, T.; Sekimoto, A.; Minato, T.; Ito, T.; Oe, Y.; Kisu, K.; Sato, E.; Funamoto, K.; Hayase, T.; Kimura, Y.; et al. reduced uterine perfusion pressure (rupp) model of preeclampsia in mice. PLoS One, 11(5):e155426, 2016.

Gonzalez-Rodriguez, P. Jr.; Tong, W.; Xue, Q.; Li, Y.; Hu, S. \& Zhang, L. Fetal hypoxia results in programming of aberrant angiotensin ii receptor expression patterns and kidney development. Int. J. Med. Sci., 10(5):532-8, 2013.

Henderson, J. T.; Thompson, J. H.; Burda, B. U.; Cantor, A.; Beil, T. \& Whitlock, E. P. Screening for Preeclampsia: A Systematic Evidence Review for the U.S. Preventive Services Task Force [Internet]. Rockville (MD), Agency for Healthcare Research and Quality (US), 2017

LeFevre, M. L. \& U.S. Preventive Services Task Force. Low-dose aspirin use for the prevention of morbidity and mortality from preeclampsia: U.S. Preventive Services Task Force recommendation statement. Ann. Intern. Med., 161(11):819-26, 2014.

Li, C.; Raikwar, N. S.; Santillan, M. K.; Santillan, D. A. \& Thomas, C. P. Aspirin inhibits expression of sFLT1 from human cytotrophoblasts induced by hypoxia, via cyclo-oxygenase 1. Placenta, 36(4):446-53, 2015.

Masuda, T.; Yano, F.; Omura, N.; Tsuboi, K.; Hoshino, M.; Yamamoto, S. R.; Akimoto, S.; Kashiwagi, H. \& Yanaga, K. Effect of Low-Dose Aspirin on Chronic Acid Reflux Esophagitis in Rats. Dig. Dis. Sci., 63(1):72-80, 2018.

Ministerio de Salud (MINSAL). Programa Nacional de la Mujer. Guía Perinatal. Santiago de Chile, Ministerio de Salud (MINSAL), Gobierno de Chile, 2015.

Nascimento-Silva, V.; Arruda, M. A.; Barja-Fidalgo, C. \& Fierro, I. M. Aspirintriggered lipoxin A4 blocks reactive oxygen species generation in endothelial cells: a novel antioxidative mechanism. Thromb. Haemost., 97(1):88-98, 2007.

Plank, C.; Nüsken, K. D.; Menendez-Castro, C.; Hartner, A.; Ostreicher, I.; Amann, K.; Baumann, P.; Peters, H.; Rascher, W. \& Dötsch, J. Intrauterine growth restriction following ligation of the uterine arteries leads to more severe glomerulosclerosis after mesangioproliferative glomerulonephritis in the offspring. Am. J. Nephrol., 32(4):287-95, 2010.

Roberge, S.; Nicolaides, K.; Demers, S.; Hyett, J.; Chaillet, N. \& Bujold, E. The role of aspirin dose on the prevention of preeclampsia and fetal growth restriction: systematic review and meta-analysis. Am. J. Obstet. Gynecol., 216(2):110-120, 2017

Sáez, O. N. \& Carvajal, C. J. Tamizaje y prevención de pre-eclampsia guiado por Doppler de arterias uterinas: revisión sistemática de la literatura. Rev. Chil. Obstet. Ginecol., 77(3):235-42, 2012.

Say, L.; Chou, D.; Gemmill, A.; Tunçalp, Ö.; Moller, A. B.; Daniels, J.; Gülmezoglu, A. M.; Temmerman, M. \& Alkema, L. Global causes of maternal death: a WHO systematic analysis. Lancet Glob. Health, 2(6):e323-33, 2014.

Steegers, E. A.; von Dadelszen, P.; Duvekot, J. J. \& Pijnenborg, R. Pre-eclampsia. Lancet., 376(9741):631-44, 2010.

Ushida, T.; Kotani, T.; Tsuda, H.; Imai, K.; Nakano, T.; Hirako, S.; Ito, Y.; Li, H.; Mano, Y.; Wang, J.; et al. Molecular hydrogen ameliorates several characteristics of preeclampsia in the Reduced Uterine Perfusion Pressure (RUPP) rat model. Free Radic. Biol. Med., 101:524-33, 2016.

Dirección para correspondencia:

Daniel Conei Valencia

Programa de Doctorado en Ciencias Morfológicas

Universidad de La Frontera

Temuco

CHILE

Email: d.conei01@ufromail.cl

Recibido : 10-02-2019

Aceptado: 22-03-2019 